\title{
THE PHOTO-ELECTRIC EARPIECE TECHNIQUE FOR RECORDING DYE DILUTION CURVES
}

\author{
BY \\ IVOR GABE* AND JOHN SHILLINGFORD $\dagger$
}

From the Postgraduate Medical School of London, W. 12

Received August 10, 1960

Stewart in 1894 first showed that it was possible to estimate the cardiac output by injecting an indicator substance into the venous circulation and sampling its concentration in the arterial circulation. Hamilton et al. (1932) demonstated its application in man and further showed that an estimation of the volume of blood through which the indicator had passed could be made from an analysis of the time-concentration curve of the indicator at the sampling site. More recently the method has been used to define the position and size of intracardiac shunts (Swan and Wood, 1953) and to give an empirical estimation of valvular regurgitation (Shillingford, 1958).

The time-concentration curves may be recorded by intermittent sampling of blood through an arterial needle, by passing arterial blood through a photo-electric cuvette, or by a photo-electric cell on the transilluminated ear. This last method has the advantage of simplicity and does not need an indwelling arterial needle, but has in the past presented certain technical problems. These have included lack of stability, sensitivity, and frequency response.

The purpose of this paper is to describe the apparatus and technique that has been developed over several years in our laboratory and to relate its practical application to the study of patients.

\section{The Photo-electric EARPIECE}

The photo-electric earpiece used to measure arterial oxygen saturation in the blood vessels of the ear (Matthes, 1936; Millikan, 1942) forms the basis of the instrument used for recording the passage of dye through the ear. It consists essentially of two photo-electric cells connected in opposition and illuminated through the ear by a small lamp. In front of one is a red or orange filter and in front of the other is an infra-red filter. Changes in ear thickness due to arterial pulsation or other causes affect both cells equally and therefore do not alter the total electrical output provided the cells are balanced. Changes in colour such as occurs with the passage of blue dye affects the cell with the red filter more than that with the infra-red and this causes a change in the total electrical output. This change in electrical potential is very small and until recently was usually recorded by a sensitive galvanometer (Wood and Geraci, 1949). The response time of such a galvanometer was rather long and tended to give damped dye dilution curves. With the development of high-stability direct current amplifiers it is now possible to amplify and record these potentials with an adequate frequency response.

The construction of a suitable earpiece presents several problems. It must be small enough to fit the pinna of the ear and this limits the size of the photo-electric cells. Selenium barrier-layer cells are the most suitable for this purpose on account of their size, but they are sensitive to changes in temperature. Norman (1959) has attempted to minimize this effect by using a cooled light source.

* Medical Research Council Fellow. 
We have found that heating the earpiece for half an hour at $40^{\circ} \mathrm{C}$. before use largely eliminates thermal drift when the earpiece is applied to the ear, and this method has the advantage that it does not increase the size of the earpiece itself.

Fig. 1 shows the design of a suitable earpiece. It consists of a small plastic casing which holds
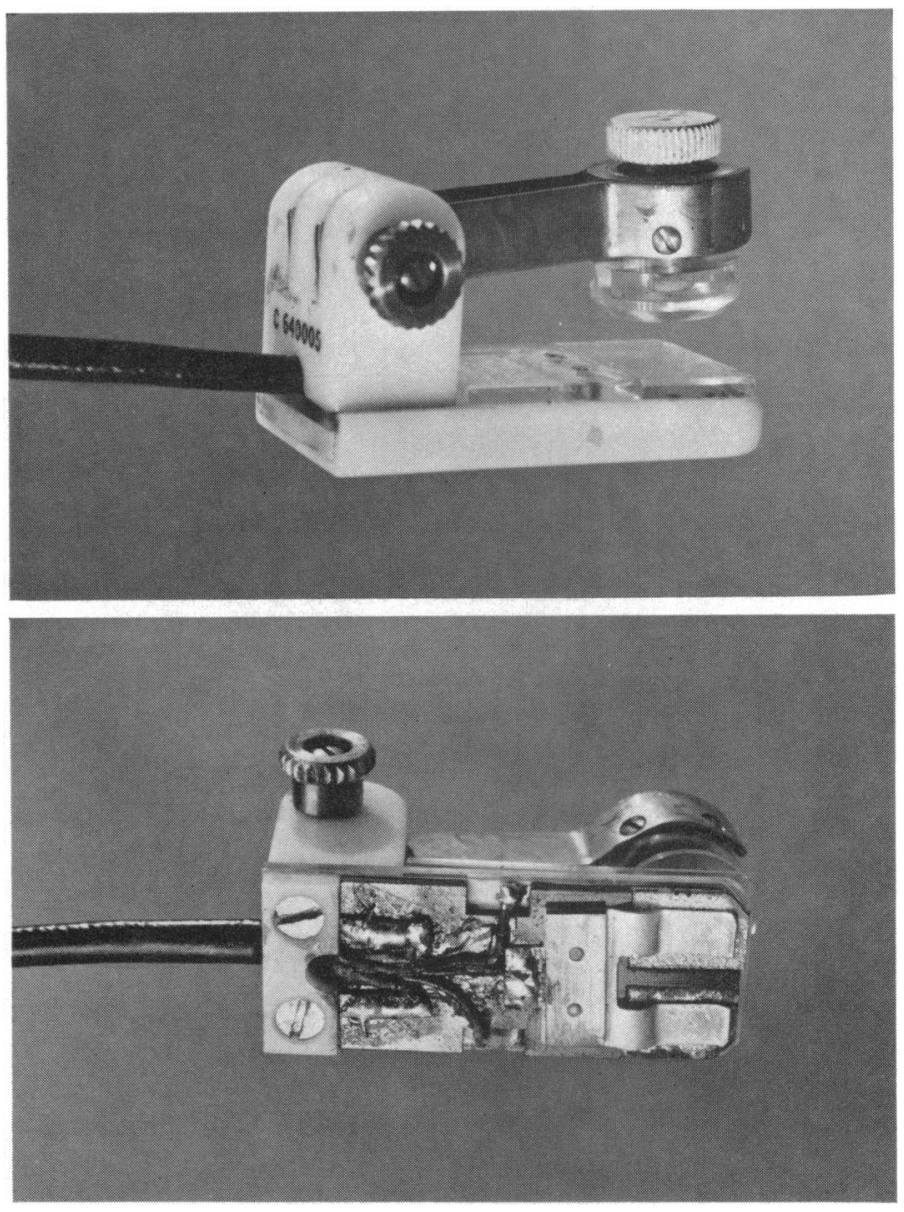

FIG. 1.-The photo-electric earpiece made by the Cambridge Instrument Company. (A) Side view. (B) Back view with cover removed.

the two photo-electric cells with their gelatin filters in front. A 3-volt 1-watt bulb enclosed in a Perspex shell is held by means of a screw clip attached to the plastic case.

\section{THE AMPLIFIER}

The output from the photo-electric cells during maximum deflection from an average dye dilution is in the order of 0.5 millivolt at an input resistance of $10,000 \mathrm{ohms}$. It is therefore necessary to use a high grade "chopper" type amplifier to obtain adequate stability. We have found the Cambridge* amplifier suitable for this purpose. Most physiological amplifiers, as found in multi-channel recording equipment, have been found to be quite unsuitable for recording dye dilution curves in that amplification and stability are inadequate.

* Cambridge Instrument Co., Ltd., Cambridge, England. 


\section{The Problem of Linearity}

If the photo-electric earpiece is to be used for quantitative work it is desirable that there should be a linear relationship between electrical output and concentration of dye in the blood. It may be profitable to consider this requirement in some detail.

It has long been established that the linearity of the relation between light intensity and current from a selenium barrier-layer photo-electric cell is greatly dependent on the value of resistance in series with the cell. Fig. 2 shows the results of an experiment in which a single earpiece cell was connected through different resistances to a Pye Scalamp galvanometer. The cell was illuminated by a 6-volt, 25-watt lamp and the intensity of illumination was varied by changing the distance of the lamp from the cell. It will be seen that over the range of light intensity used, a linear relation between current and illumination existed only when the resistance in series with the photocell was $4830 \mathrm{ohms}$ or less. Under these circumstances, in accordance with Beer's law of optical absorption, an exponential relationship would exist between current and the concentration of a dye solution placed between the photocell and the lamp. We may see that this expectation is borne out very nearly by arranging a cuvette (Fig. 3) between the ear and complete earpiece and measuring the output of the amplifier (input resistance $4700 \mathrm{ohms}$ ) when the concentration of aqueous Coomassie Blue in the cuvette is varied. The results of such an experiment are shown in Fig. 4. On completion of the set of readings the cuvette was filled with water and an injection of Coomassie Blue was given intravenously. The galvanometer deflection produced three minutes after the injection was noted, the plasma concentration at this time being $13.0 \mathrm{mg}$. $/ 1$. By this method the comparative effects of dye in the blood and in the cuvette may be estimated.

From Fig. 4 it can be seen that the desired linear relation between concentration of dye and photocell output does not exist. However, the approximation may reasonably be made that for small changes in concentration only, the relation may be taken as linear. This means that if

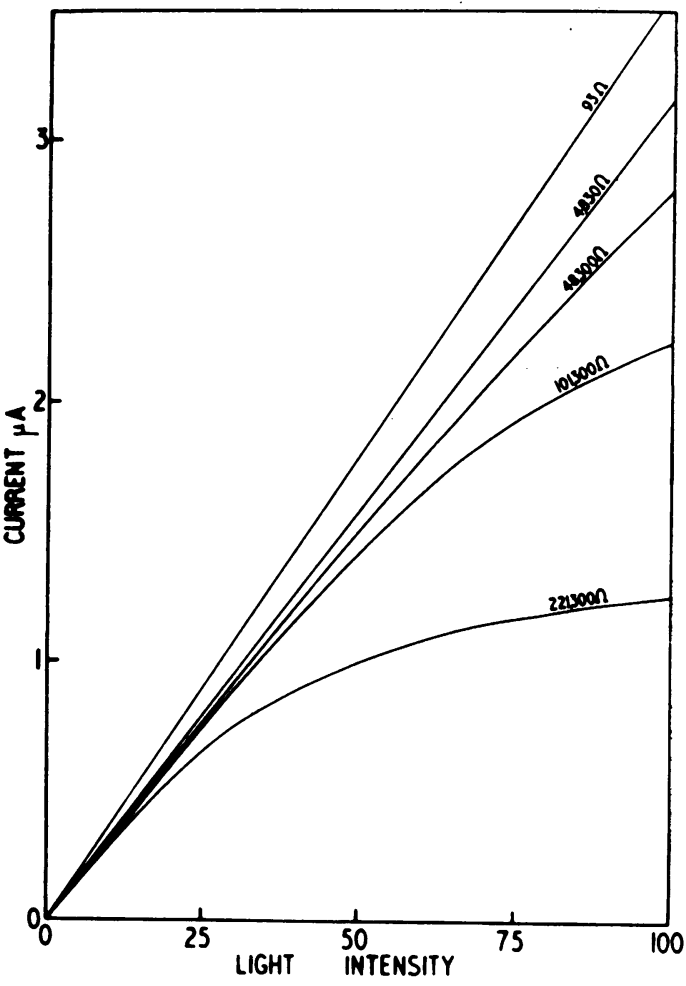

FIG. 2.-The relationship between intensity of illumination and current output from a single selenium barrier-layer cell (size $5 \mathrm{~mm} . \times 9 \mathrm{~mm}$.), showing the effect of different external resistances. The units of light intensity are arbitrary but it was arranged that the maximum light intensity (100 on the scale) was approximately the same as that on the photocell when it was fitted to an earpiece and illuminated through an ear by a 3-volt, 1-watt bulb.

the dye curve is to be calibrated from the height of the "tail" of the curve, care must be taken not to give too large a dose of dye; and that the sensitivity of the instrument will change as the basal level of dye in the blood rises.

If it were possible to extend the range of effective linearity it would be legitimate to give larger doses of dye. It would also be possible to make estimates of relative cardiac outputs in one individual by giving successive injections of the same quantity of dye and merely measuring the area beneath each dye curve, no calibration being necessary: the restrictive assumption is that the amount of blood in the light pathway is unchanged during the procedure.

The range of linearity may be extended by using the fact that when a barrier-layer photocell is connected to a high resistance the relation between current output and light intensity is alinear, 


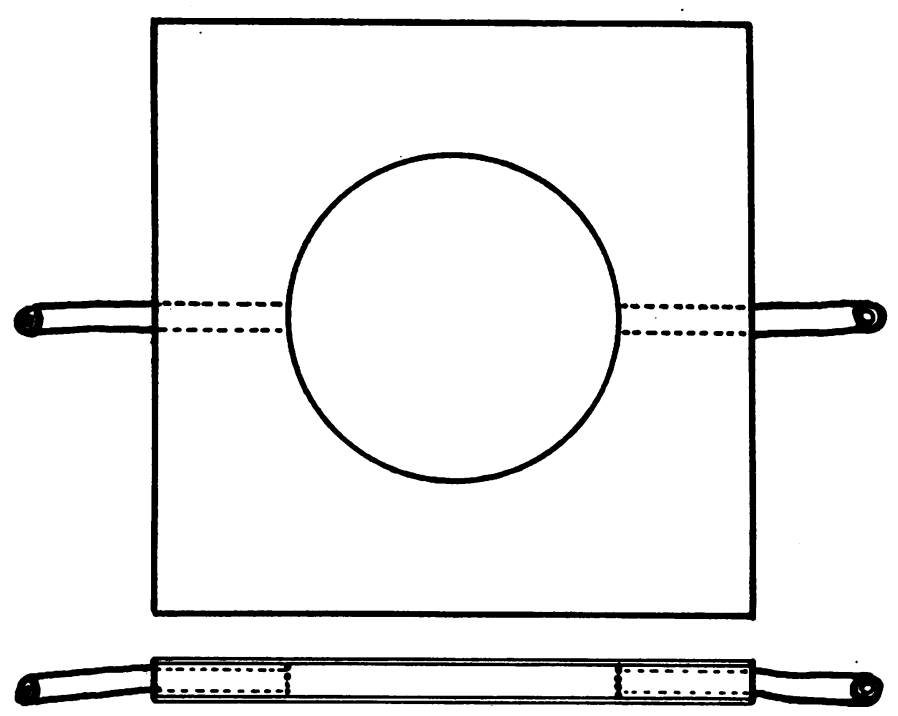

FIG. 3.-The cuvette used for testing linearity. It consists of two microscope cover slips cemented to a Perspex spacer $1 \mathrm{~mm}$. thick, forming a chamber which can be filled with calibrating solutions through polythene tubing. The cuvette is fixed to the earpiece between the light and photocells by adhesive tape for in vitro tests, and it is small enough for the ear to be included in the light pathway as well if necessary.

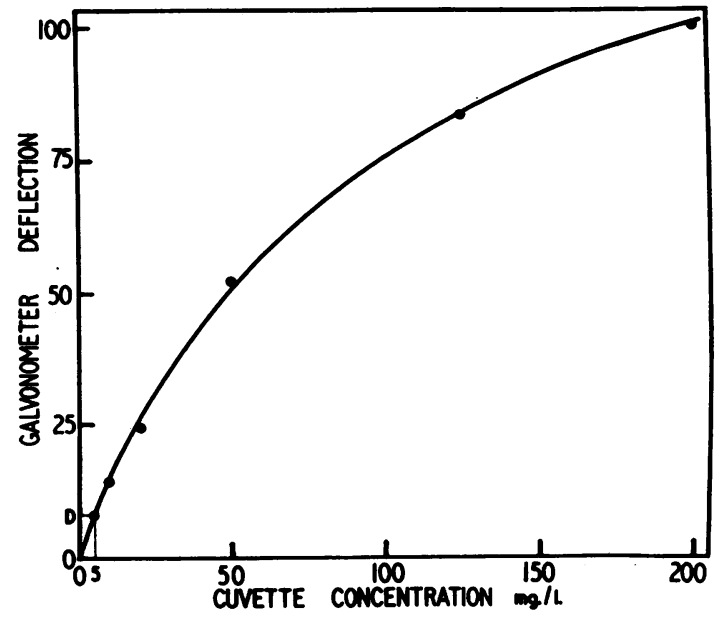

FIG. 4.-The relation between galvanometer deflection and concentration of aqueous Coomassie Blue in a cuvette applied to the ear. The earpiece was connected to an amplifier with an input resistance of 4700 ohms with respect to the "red" cell. The vertical scale is arbitrary. The galvanometer deflection $\mathrm{D}$ was that produced three minutes after an intravenous injection of Coomassie Blue, when the plasma concentration was $13 \cdot 0 / 1$.

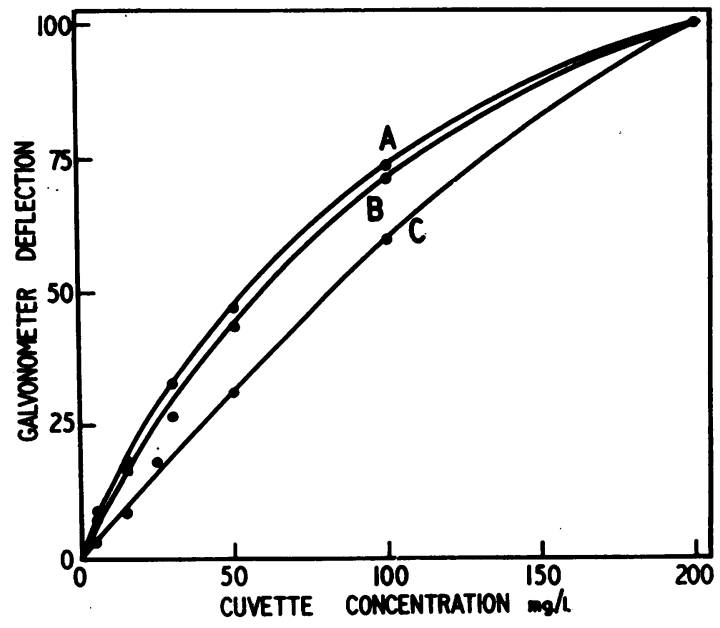

Fig. 5.-Concentration of aqueous Coomassie Blue in the cuvette against deflection of a Pye Scalamp galvanometer. The "red" photocell was connected to the galvanometer through an external resistance: (A) $560 \mathrm{ohms}$, (B) $10,000 \mathrm{ohms}$, (C) 470,000 ohms. The vertical scale is arbitrary and the three curves have been plotted to the same final value to make comparison easier.

as shown in Fig. 2; the current output is proportional to the logarithm of light intensity. This alinearity tends to oppose to the alinearity implicit in Beer's Law. This property has been used in oximetry to produce an instrument that records oxygen saturation linearly (Goldie, 1942; Paul, 1953). Experimentally we have found the effect can be used to produce near-linearity between current output and concentration of dye over a wide range.

Fig. 5 shows concentration of aqueous Coomassie Blue in the cuvette against deflection of a 


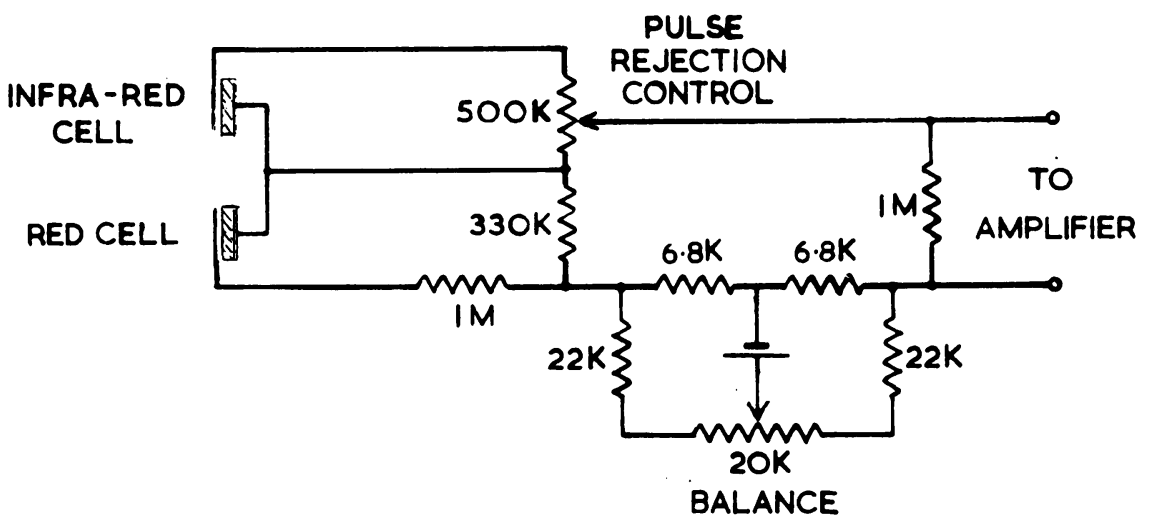

FIg. 6.-The input circuit. (See text).

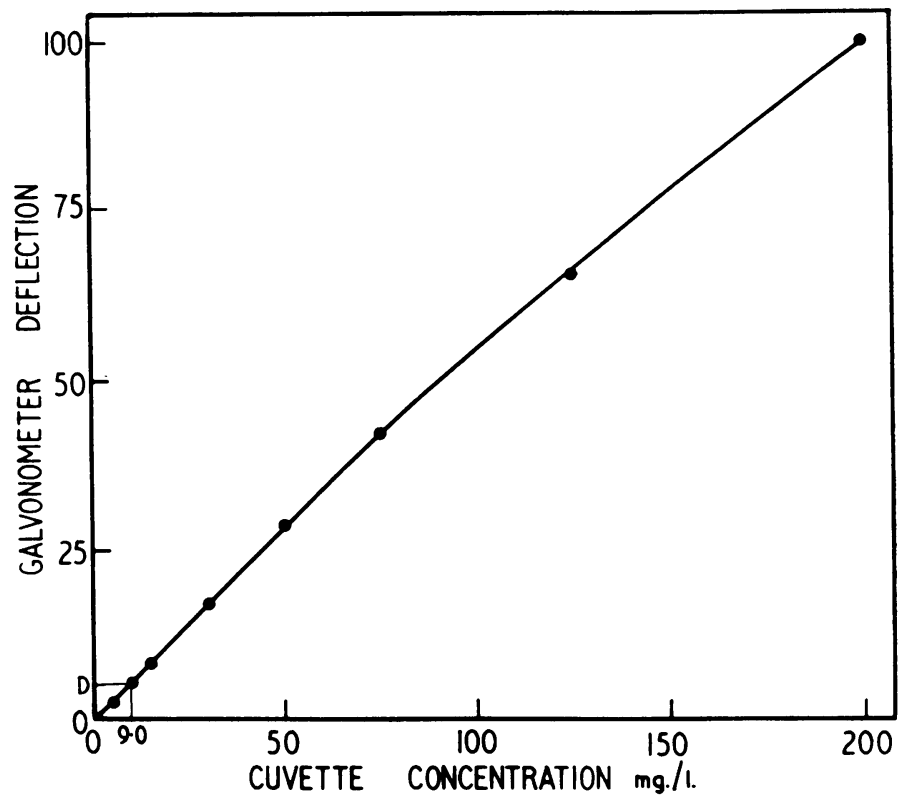

Fig. 7.-Galvanometer deflection against cuvette concentration of aqueous Coomassie Blue with the earpiece applied to the ear. The input circuit was that shown in Fig. 6. The vertical scale is arbitrary. The galvanometer deflection $\mathrm{D}$ was that produced three minutes after an intravenous injection of Coomassie Blue, when the plasma concentration was $18 \cdot 2 \mathrm{mg} . / 1$.

Pye Scalamp galvanometer produced directly by the "red" photocell. Readings were taken when the effective resistance across the photocell was $560,10,000$, and $470,000 \mathrm{ohms}$. For practical purposes the curve in which the external resistance was $470,000 \mathrm{ohms}$ can be regarded as linear up to a cuvette concentration of about $100 \mathrm{mg}$./1., while the other two curves are showing obvious alinearity before a concentration of $30 \mathrm{mg} . / 1$. is reached.

In this laboratory we now connect 1.33 megohms in series with the red cell and 0.5 megohms in series with the infra-red cell (Fig. 6). A calibration curve showing this input arrangement is shown in Fig. 7. Injection of a quantity of Coomassie Blue intravenously revealed that $18.2 \mathrm{mg} . / \mathrm{l}$. of 
the dye in the plasma produced the same electrical response as $9.0 \mathrm{mg}$./l. of dye in the cuvette. We should expect this system to be linear for practical purposes up to a plasma concentration of about $150 \mathrm{mg} . / 1$.

Further evidence on the linearity of this apparatus was obtained by giving four successive injections at eight-minute intervals of $50 \mathrm{mg}$. Coomassie Blue to four normal subjects. If the apparatus is linear and there is no change in ear thickness, the area of paper beneath the dye curve (excluding the effects of recirculation by extrapolation) should be inversely proportional to the cardiac output in each subject. As the subjects were resting calmly during the experiment, we should expect little evidence of any change in cardiac output. A steady rise in estimated cardiac output would be consistent with alinearity of the apparatus. Fig. 8 shows the four dye curves obtained in one subject (J.B.) and Table I gives the collected results from all the subjects.

\section{THE RECORDER}

The curves may be recorded either by a photographic recorder or a direct-writing instrument. The paper width should be at least $7 \mathrm{~cm}$. and a convenient paper speed is 6 inches a minute. For

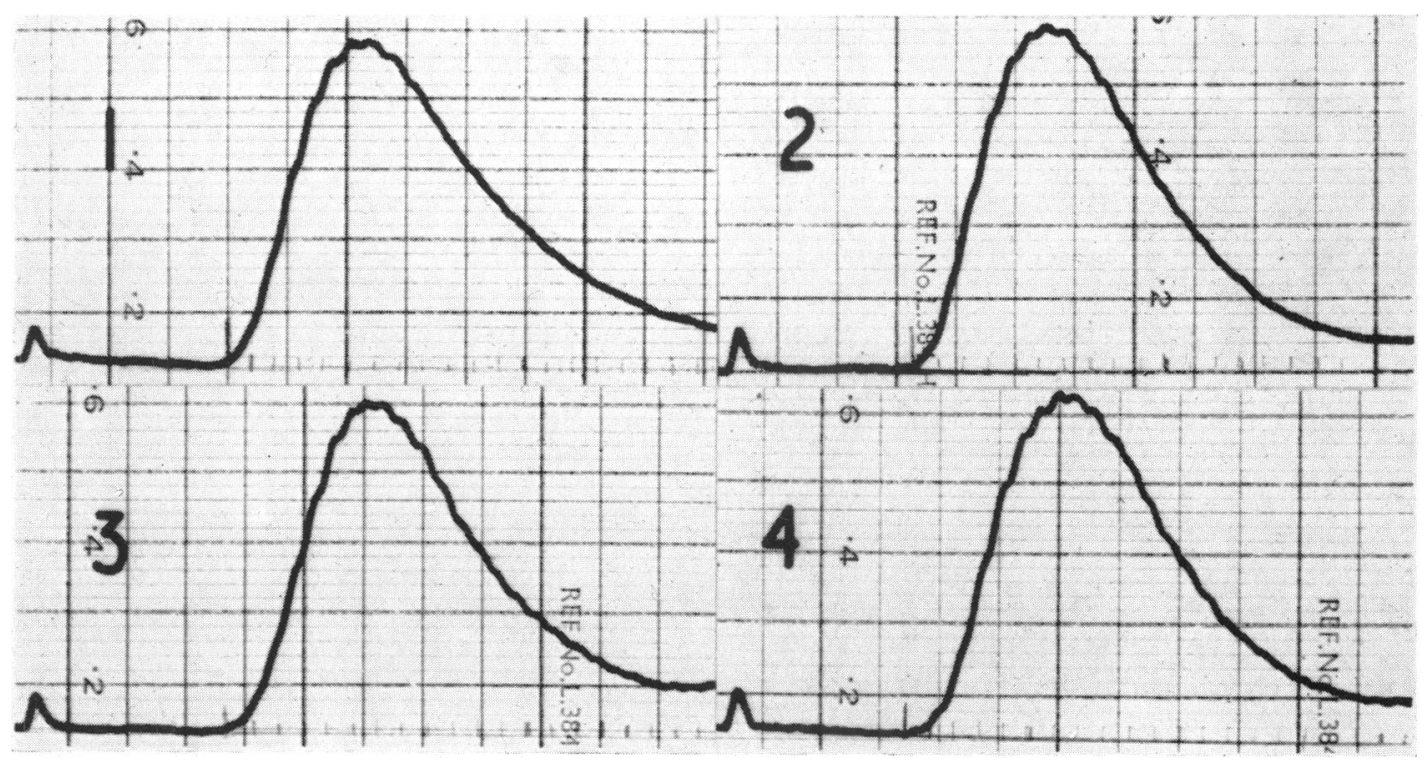

FIG. 8.-Four dye curves obtained when a normal subject (J.B.) was given four successive injections of $50 \mathrm{mg}$. Coomassie Blue at eight-minute intervals.

TABLE I

Successive Estimations of Cardiac Output in Normal Resting Subjects, Expressed as Proportions of the INITIAL DETERMINATIONS

\begin{tabular}{c|c|c|c|c}
\hline & \multicolumn{4}{|c}{ Order of Estimation } \\
\cline { 2 - 5 } Subject & 1 & 2 & 3 & 4 \\
\hline J.B. & 1.00 & 1.04 & 1.01 & 1.02 \\
A.B. & 1.00 & 1.08 & 0.98 & 1.10 \\
F.G. & 1.00 & 1.12 & 1.03 & 1.05 \\
H.A. & 1.00 & 0.99 & 1.00 & 1.01 \\
\hline
\end{tabular}


most purposes a response time for full-scale deflection of 2 seconds is adequate although for infants, where the circulation is very rapid, a faster response time may be desirable. We have found either an Evershed and Vignole one milliampère, $3000 \mathrm{ohm}$ pen recorder, or the Cambridge Instrument Company's photographic or servo direct-writing recorders satisfactory.

\section{Comparison of Earpiece and CuvetTe CuRves}

Lacy et al. (1957) have studied the distorting effects of the sampling system when dye dilution curves are made by passing arterial blood through polythene tubing to a photo-electric cuvette. Distortion could be made negligible by increasing the flow of blood or decreasing the length or the diameter of the tubing. It would be expected that the ear used as a "cuvette" would also be capable of distorting the time-concentration pattern of dye in the arterial system. However, there are reasons for supposing that distortion so produced is small enough to be neglected for most purposes, unless the duration of the curve is very short.

Any comparison between dye dilution curves made by an arterial cuvette and an earpiece should be recorded by equipment with an adequate frequency response. Fig. 9 shows two dye dilution

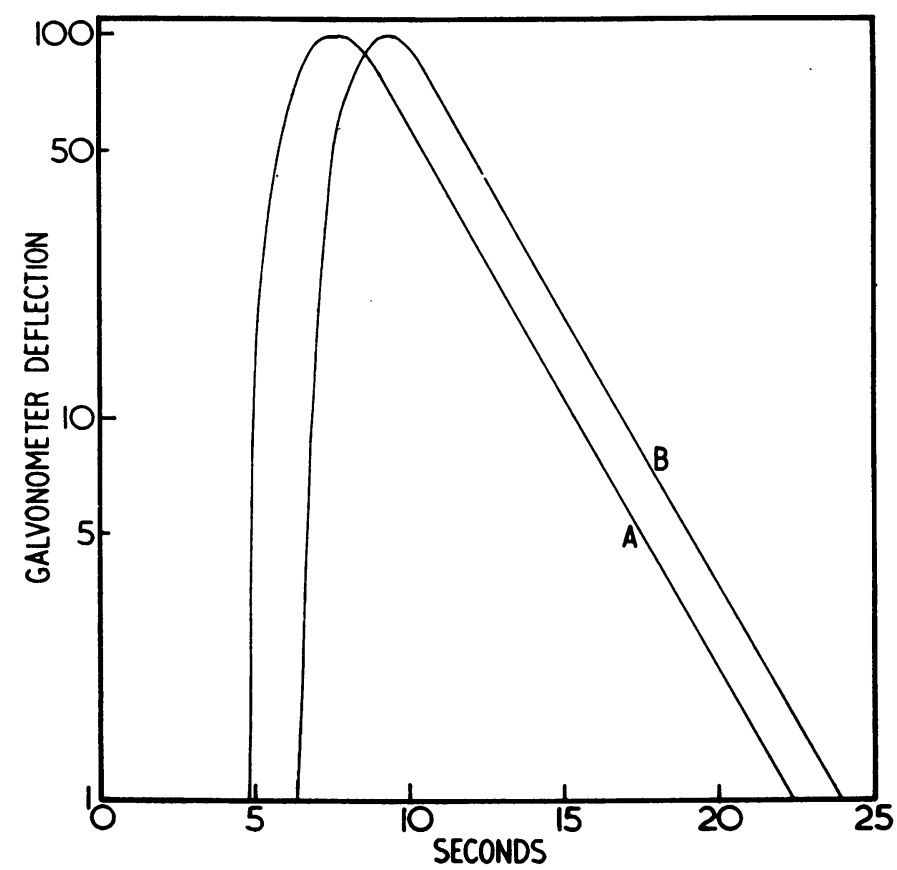

FIG. 9.-Two dye dilution curves made by injecting Coomassie Blue into the pulmonary artery of an 11-year-old boy whose ventricular septal defect had previously been closed. The curves have been plotted semi-logarithmically and extrapolated. The peak heights have been made the same. Curve $A$ is from a photo-electric earpiece connected to the input circuit shown in Fig. 6. Curve B was made by passing blood from the femoral artery through a Cambridge photo-electric cuvette. The length of tubing involved was $15 \mathrm{~cm}$. and its internal diameter $1.2 \mathrm{~mm}$. Blood flowed through the tubing at $0.8 \mathrm{ml} . / \mathrm{sec}$. (See text).

curves replotted semilogarithmically. Curve $A$ was from an earpiece and was made within three minutes of Curve B, which was made by passing blood from the femoral artery through a photoelectric cuvette. The peak height of the curves has been made the same to allow for differences in sensitivity of the two systems. The area beneath Curve A (extrapolated to infinity) exceeds that 
beneath curve $B$ by only 5 per cent. The slope of A is $0.326 \mathrm{sec}^{1}$ and that of $\mathrm{B} 0.328 \mathrm{sec}^{-1}$, the disagreement being insignificant. The main difference between the two curves is due merely to the difference in distance between the heart and the two sampling sites.

\section{INDICATOR DYES}

Many dyes have been used in the study of the circulation in man. An ideal indicator should meet the following requirements.

(1) It should be harmless when injected intravenously and cause no discomfort.

(2) The amount injected should be small in bulk.

(3) The indicator must remain in the blood stream and not be lost in the tissues or changed in chemical composition during its passage through the body.

(4) It must be capable of being easily estimated in blood samples.

Until recently Evans Blue was commonly used for obtaining dye dilution curves. The loss of this dye is small over the time needed to calibrate the curve; it appears to be harmless and its concentration can be measured by extracting the dye from the plasma. Its absorption spectrum (maximal at 620 millimicrons), however, is in the region of that of reduced hæmoglobin; it is therefore often not satisfactory in those patients with cyanotic heart disease and fluctuating arterial oxygen saturation. Moreover, it is only slowly excreted and stains the skin when given in large amounts. To overcome this Fox et al. (1957) introduced a green dye (Cardio-green) with maximal absorption at a wavelength in the region of 800 millimicrons, at which absorption by oxy- and reduced hæmoglobin is the same. This dye, although satisfactory for use with a cuvette, may involve undue arterial pulsation when used with an earpiece.

More recently Coomassie Blue (Imperial Chemical Industries) (Taylor and Shillingford, 1959; Taylor and Thorp, 1959) has been found to offer several distinct advantages in that it does not stain the tissues and is excreted comparatively rapidly. The effects of changing oxygen saturation in the blood can be minimized by reducing the sensitivity of the recording instrument and giving larger doses of the dye. The average dose is between 0.2 and $0.8 \mathrm{mg}$./kilo and we have not observed toxic effects up to a total dose of $3 \mathrm{mg}$./kilo. In larger doses vomiting and vaso-vagal disturbances may occur, but we have not found it necessary to exceed a single dose of more than $0.8 \mathrm{mg}$./kilo to obtain acceptable curves. When using this dye for measuring cardiac output and calibrating the curve from the height of the tail at a known blood concentration, errors may be introduced by the somewhat rapid fall in blood level over the first three minutes.

Other dyes, such as indigo-carmine and methylene blue, have been used with success for qualitative observations, particularly for the definition of intracardiac shunts, but rapid excretion precludes their use for any quantitative work.

The technique for the estimation in blood of Evans Blue has been described by Murray and Shillingford (1958) and for the estimation of Coomassie Blue by Taylor and Shillingford (1959).

\section{The Injection Syringe}

It is difficult to measure either Evans Blue or Coomassie Blue dye accurately without a special syringe. We have used two types with success. One is an ordinary 5-ml. Luer-lock syringe with a scratch made on the barrel $1 \mathrm{~cm}$. behind the tip: the dye can then be drawn up into the syringe and the scratch aligned against a similar marking on the syringe. An alternative syringe for multiple identical injections is shown in Fig. 10. It is made from a 10-ml. Luer-lock syringe, around the barrel of which is cemented a metal ring into which are screwed a series of stops of different lengths: a second ring and stop is cemented to the piston, and the difference of length between each adjacent stop determines the travel of the piston.

\section{Determination of the Quantity of Dye Injected}

For estimation of cardiac output it is necessary to know the exact quantity of dye injected through the needle or catheter. This is done by taking the needle or catheter at the end of the 


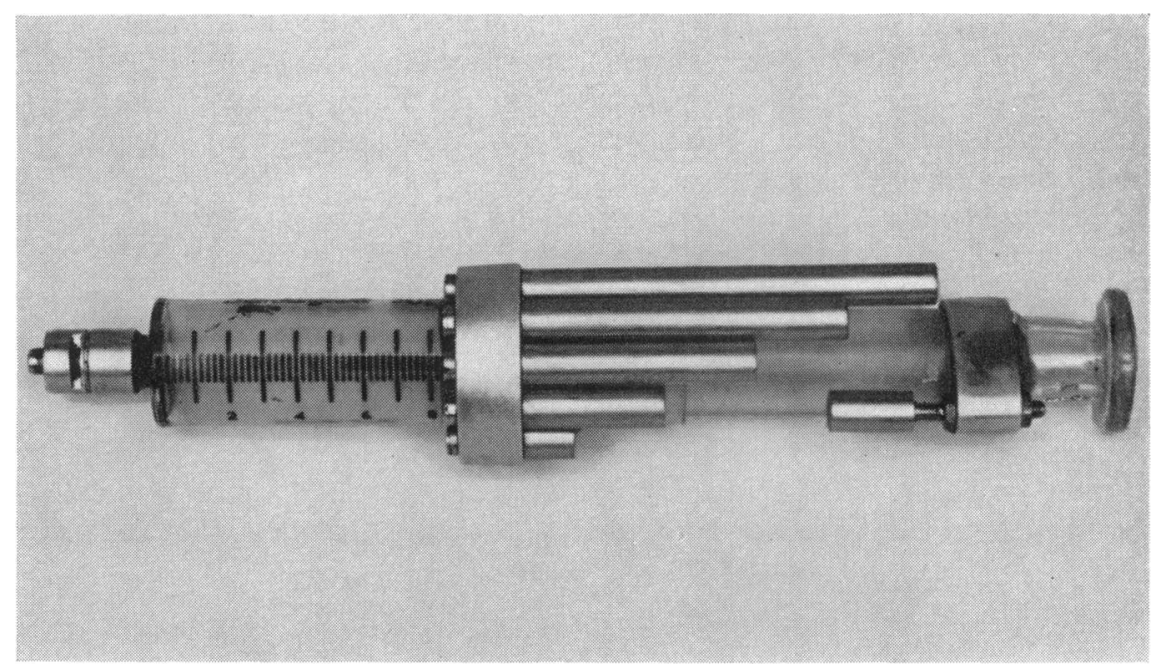

Fig. 10.-Injection syringe designed to deliver four equal volumes of dye solution.

procedure, washing, and drying the lumen by passing a stream of air or oxygen through it. The syringe is filled with water to the mark used at the time of injection and the empty needle or catheter attached. The water is then ejected into a weighed receptacle and the weight again determined. The whole procedure is repeated three times and the average of the three readings used for calculations. This procedure allows for the "dead space" in the catheter or needle.

\section{The Earpiece Heater}

To achieve adequate stability the earpiece must reach thermal equilibrium. On account of this it is desirable, especially in cold weather, to preheat the earpiece to approximately body heat. A simple heater can be made by winding a short length of resistance wire (approximately 8 ohms) around a small metal cannister between two layers of adhesive tape and connecting it to a 6-volt supply. The earpiece is placed in this an hour before use.

\section{Preparation of the Patient}

The injection may be given intravenously or at the time of cardiac catheterization. If it is given intravenously, a medial arm vein should be used as a lateral vein injection often produced distorted curves because of uneven venous flow into the right atrium. The room and the patient should be warm, for vasoconstriction due to cold surroundings must be avoided. Better results are often obtained with intravenous injections when the patient is sitting up or tilted feet down.

The ear should be warm and further vaso-dilatation may be obtained by rubbing in histamine cream on each side of the pinna. The earpiece is placed on the ear and secured in position by pieces of adhesive tape. The lamp should just rest on the ear, which should not be compressed. The lead from the earpiece should be fixed to the head with adhesive tape to prevent movement of the earpiece. The ear should be covered by a small black cloth to exclude extraneous light. The earpiece must remain on the ear with the lamp alight for at least fifteen minutes before use. If there is considerable baseline fluctuation, due to a changing blood oxygen saturation, this may sometimes be improved by allowing the patient to breathe oxygen through a plastic mask. The input circuit of the amplifier allows for pulse rejection and the control should be adjusted for minimal pulsation. The amount of dye injected and the position of the sensitivity control on the machine are a matter of trial and error. In general, a good curve should be drawn in adults with a $20 \mathrm{mg}$. dose of either Coomassie Blue or Evans Blue and the sensitivity of the machine adjusted accordingly after the 
initial injection. If the earpiece has been correctly applied, the baseline in non-cyanotic patients should be reasonably flat. In cyanotic patients, however, with considerable variation in oxygen saturation, it may be necessary to reduce the gain of the amplifier and increase the dose two or three times in the case of Coomassie Blue. This does not apply to Cardio-green, in which only the infrared cell is used for recording the passage of the dye.

It may not in occasional cases be possible to record the passage of dye through the ears of babies or very young children, possibly because of a poor arterial supply at this age. In other patients a rhythmic slow oscillation of the baseline at about the respiratory rate may be noticed and continues even if the patient holds his breath; this change is not usually serious enough to detract from the accuracy of diagnosis. Baseline drift should not occur if the amplifier and earpiece have been allowed to warm for an hour and the latter applied to the ear for at least fifteen minutes. If serious instability is experienced, the earpiece should be removed from the ear and a piece of paper inserted between the lamp and cells. If the instability continues, the trouble may be in the lamp or its connections, which should be checked; if these are in order the earpiece cells or the amplifier are at fault.

\section{SUMMARY}

The principles governing the recording of dye dilution curves by the photoelectric earpiece are briefly described. The associated apparatus and the problem of linearity is considered, and a way in which the range of linearity may be extended is discussed. The practical technique of dye dilution curves made by the photo-electric earpiece is given.

\section{REFERENCES}

Fox, I. J., Brooker, L. G. S., Heseltine, D. W., Essex, H. E., and Wood, E. H. (1957). Proc. Mayo Clinic., 32, 478. Goldie, E. A. G. (1942). J. Sci. Instrum., 19, 23.

Hamilton, W. F., Moore, J. W., Kinsman, J. M., and Spurling, R. G. (1932). Amer. J. Physiol., 99, 534.

Lacy, W. W., Emanuel, R. W., and Newman, E. V. (1957). Circulat. Res., 5, 568.

Matthes, K. (1936). Arch. exp. Path. Pharmak., 181, 630.

Millikan, G. A. (1942). Rev. Sci. Instrum., 13, 434.

Murray, J. F., and Shillingford, J. P. (1958). J. clin. Path., 11, 170.

Norman, J. (1959). Brit. Heart J., 21, 107.

Paul, W. (1953). J. Sci. Instrum., 30, 165.

Shillingford, J. P. (1958). Brit. Heart J., 20, 229.

Stewart, G. N. (1894). J. Physiol., 15, 1.

Swan, H. J. C., and Wood, E. H. (1953). Proc. Mayo Clinic., 28, 95.

Taylor, S. H., and Shillingford, J. P. (1959). Brit. Heart J., 21, 497.

Taylor, and Thorp, J. M. (1959). Brit. Heart J., 21, 492.

Wood, E. H., and Geraci, J. E. (1949). J. Lab. clin. Med., 34, 387. 